Research Paper

\title{
Development and Validation of a Nomogram to Predict the Benefit of Adjuvant Radiotherapy for Patients with Resected Gastric Cancer
}

\author{
Shu-Qiang Yuan ${ }^{* *}$, Wen-Jing Wu2,3*, Miao-Zhen Qiu, ${ }^{4 *}$, Zi-Xian Wang4*, Lu-Ping Yang4, Ying Jin ${ }^{4}$

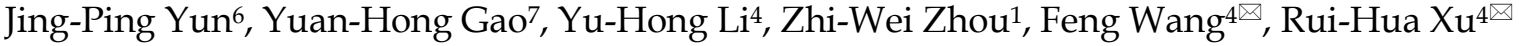

1. Department of Gastric Surgery, Sun Yat-sen University Cancer Center; State Key Laboratory of Oncology in South China; Collaborative Innovation Center for Cancer Medicine, Guangzhou, 510060, China;

2. Guangdong Provincial Key Laboratory of Malignant Tumor Epigenetics and Gene Regulation, Medical Research Center, Sun Yat-sen University Memorial Hospital, Guangzhou, 510120, China;

3. Department of Breast Oncology, Sun Yat-sen University Memorial Hospital, Guangzhou, 510120, China;

4. Department of Medical Oncology, Sun Yat-sen University Cancer Center; State Key Laboratory of Oncology in South China; Collaborative Innovation Center for Cancer Medicine, Guangzhou, 510060, China;

5. Department of Pathology, Johns Hopkins University School of Medicine, Baltimore, MD 21231, USA;

6. Department of Pathology, Sun Yat-sen University Cancer Center; State Key Laboratory of Oncology in South China; Collaborative Innovation Center for Cancer Medicine, Guangzhou, 510060, China;

7. Department of Radiotherapy, Sun Yat-sen University Cancer Center; State Key Laboratory of Oncology in South China; Collaborative Innovation Center for Cancer Medicine, Guangzhou, 510060, China.

*These authors contributed equally to this study.

$\square$ Corresponding author: Rui-Hua Xu, Department of Medical Oncology; Sun Yat-Sen University Cancer Center, 651 Dong Feng Road East, Guangzhou 510060, China; Tel and Fax: +86-20-8734 3468; E-mail: xurh@sysucc.org.cn; Feng Wang, Department of Medical Oncology; Sun Yat-Sen University Cancer Center, 651 Dong Feng Road East, Guangzhou 510060, China; Tel and Fax: +86-20-8734 3795; E-mail: wangfeng@sysucc.org.cn.

(1) Ivyspring International Publisher. This is an open access article distributed under the terms of the Creative Commons Attribution (CC BY-NC) license (https://creativecommons.org/licenses/by-nc/4.0/). See http://ivyspring.com/terms for full terms and conditions.

Received: 2017.03.01; Accepted: 2017.09.07; Published: 2017.09.29

\begin{abstract}
Background: The US guidelines for gastric cancer (GC) recommend adjuvant radiotherapy (ART) combined with 5-fluorouracil as a standard treatment for patients with resected locally advanced GC. However, patient selection criteria for optimizing the use of adjuvant therapies are lacking. In this study, we developed and validated a nomogram to predict the individualized overall survival (OS) benefit of ART among patients with resected $\geq$ stage IB GC.

Patients and Methods: The 2002-2006 Surveillance, Epidemiology, and End Results (SEER) data of 5,206 patients with resected GC were used as a training set for the development of a nomogram. The 2007-2008 SEER data of 1,986 patients with resected GC were used as validation data.

Results: In the multivariate analysis weighted by inverse propensity score, the efficacy of ART varied by the ratio of positive to examined nodes $\left(P_{\text {interaction }}<0.01\right)$. The magnitude of this difference was included in the nomogram with associated prognosticators to predict the 3-and 5-year OS with and without ART. The nomogram showed significant prognostic superiority to the $8^{\text {th }}$ TNM staging in the training set (Concordance index, 0.68 versus $0.65 ; P<0.01$ ) and the validation set (Concordance index, 0.68 versus $0.64 ; P<0.01$ ). Moreover, the calibration was accurate, and the actual efficacy of ART was positively correlated with the nomogram-estimated survival benefit from ART ( $P_{\text {interaction }}<0.01$ and $P_{\text {interaction }}=0.02$ in the training set and the validation set, respectively).

Conclusion: The nomogram can aid individualized clinical decision making by estimating the 3and 5-year OS and potential benefits of ART among patients with resected GC.
\end{abstract}

Key words: gastric cancer; adjuvant radiotherapy; survival; nomogram; Surveillance, Epidemiology, and End Results (SEER). 


\section{Introduction}

Gastric cancer (GC) is the fifth most common malignancy and the third leading cause of cancer-related death worldwide, with an especially high incidence in East Asia [1-3]. Currently, patients with resectable locally advanced tumors have high rates of postsurgical recurrence and a dismal prognosis, which has prompted efforts to improve outcomes via chemotherapy and radiation [4-7].

The landmark Intergroup 0116 trial demonstrated that adjuvant radiotherapy (ART) combined with fluorouracil/leucovorin provided significant disease-free survival and overall survival (OS) benefits for patients with resected locally advanced GC compared with surgery alone. [4] Accordingly, for patients with locally advanced GC undergoing curative surgery, the National Comprehensive Cancer Network guidelines recommend adjuvant chemoradiotherapy as a standard treatment for patients with radically resected GC [8]. However, we lack effective risk-stratified tools to optimize the use of adjuvant chemoradiotherapy by improving survival probabilities in selected individuals and avoiding overtreatment in others. Although several good nomograms have been created to refine the survival prediction for patients with resected GC [9-11], none of them were tested the abilities to guide patient selection for adjuvant treatments.

In this study, we developed a nomogram to predict the 3- and 5-year OS with and without ART for patients with resected GC using a population-based cohort from the Surveillance, Epidemiology, and End Results (SEER) database. The aim of this study was to develop a practical clinical tool that could be used for individualized risk assessment and to guide individualized management decisions in the use of ART.

\section{Patients and Methods}

\section{Data sets}

Using the SEER database (18 registries), we identified 12,442 patients 18 years or older with GC that was diagnosed from January 2002, to December 2008. We selected this time range to reflect the contemporary practice of ART in the US because the results of the Intergroup 0116 trial, which is the landmark study for the use of ART, were published in September 2001 [4]. This time range also allowed for a longer median follow-up period to reflect the long-term survival in this cohort. The inclusion criteria were as follows: (1) microscopically confirmed single primary GC (ICD-O-3 codes: $8010-8231$ and
8255-8576); (2) gastrectomy with at least one node harvested; and (3) active follow-up. Patients with carcinoma in situ (63 cases), distant metastasis or unknown status of metastasis (1,781 cases), preoperative radiotherapy, unknown radiation sequence with surgery, and non-beam radiation (271 cases) were excluded. Patients who survived $<6$ months after their initial diagnosis (1,543 cases) were excluded to account for the immortal time bias. Stage information was created according to the $8^{\text {th }}$ American Joint Committee on Cancer (AJCC) TNM classification, and those with insufficient information for conversion to $8^{\text {th }}$ AJCC staging were excluded (76 cases). Those with Stage IA tumors (1,516 cases) were excluded because of the rare use of ART among these patients. The final analytic SEER cohort consisted of 7,192 patients. No institutional review was sought because SEER is public-use data.

The data of 5,206 patients in the SEER cohort diagnosed from January 2002 to December 2006 in the SEER cohort were used as a training set for the development of a nomogram and accompanying Web software. The data of 1,986 patients diagnosed from January 2007, to December 2008, were used as validation data because the results of the MAGIC trial, which provided solid evidence for the use of perioperative chemotherapy as an alternative standard treatment without including irradiation in resected GC, were published in July 2006 [5]. The performance of the proposed nomogram in patients diagnosed after 2006 would clarify whether the nomogram is clinically useful under the contemporary treatment modality of GC.

\section{Statistical analysis}

To infer missing data on variables in the SEER cohort (race, tumor location, size, and differentiation), we applied multiple imputations using missForest (Supplementary Table 1) [12].

We classified patients in the SEER cohort with the radiation code of "beam radiation" into the surgery+ART group (ART group) and those with the codes of "none" and "refused" into the non-ART group. Inverse probability propensity score weighting [13] was used to balance patient characteristics between the ART and non-ART groups in the training set and the validation set.

OS was the primary outcome of interest. Multivariate Cox regression models with robust sandwich variance estimators were used to assess the relationships between covariates and OS in the weighted samples [14]. Multivariable fractional polynomial interactions were used to handle the interactions of ART with continuous variables [15]. For model construction, we began with the receipt of 
ART, other accounted variables (patient age, year of diagnosis, race, tumor location, size, differentiation, $\mathrm{T}$ stage, and lymph node ratio [16] (LNR, ratio of positive to total examined nodes)), and their first-order interaction terms. The final Cox models were obtained using backward stepwise selection of the variables $(P<0.05)$.

A nomogram was developed to predict the 3and 5-year OS probabilities, given the associated risk factors. Concordance indices (C-indices) were used to compare the discriminative abilities of the nomogram and $8^{\text {th }}$ AJCC TNM classification (i.e. the model including the $8^{\text {th }}$ AJCC $\mathrm{T}$ and $\mathrm{N}$ classifications). [17] Calibration was performed by reviewing the plots of nomogram-predicted survival probabilities with the Kaplan-Meier-estimated probabilities. [18] Bootstraps with 1,000 resamples were used to quantify model overfit and calculate Kaplan-Meier estimates. External validation of the nomogram was performed using the SEER validation set.

Statistical significance was set as $P<0.05$ in a two-tailed test. The statistical analyses were performed using SAS v.9.3 (SAS Institute, Cary, NC, USA), SPSS v.19.0 (SPSS, Chicago, IL, USA), and R v.3.2.3 (http:/ / www.r-project.org).

The statistical methods are further detailed in the

\section{Supplementary Methods.}

\section{Results}

The clinicopathologic characteristics for the training set (5,206 cases) and the validation set $(1,986$ cases) are listed in Table 1 . The percentages of patients treated with ART were similar in the training set and the validation set $(47.1 \%$ vs. $47.0 \%)$. Balance in the patient characteristics between the ART and non-ART groups was achieved after multiple imputations and inverse propensity score weighting among both datasets (Supplementary Tables 2-3). Kaplan-Meier OS estimates were $35.3 \% \quad(95 \%$ confidence interval [CI]: $34.6-36.0 \%)$ and $38.2 \%(95 \%$ CI: $37.1-39.3 \%)$ in the training set and the validation set, respectively.

In the training set weighted by inverse propensity score, the final Cox model was obtained by stepwise backward selection (Table 2). The only treatment interaction term retained in the final model was with LNR $\left(P_{\text {interaction }}<0.01\right)$, suggesting that the effect size of ART varied according to LNR; that is, ART was significantly associated with a greater than $20 \%$ reduction of risk of death in patients with a higher LNR (LNR>0.2, the median value), whereas OS was similar between the treatment groups in those with a lower $\mathrm{LNR}(\mathrm{LNR} \leq 0.2$; Figure 1).
Table 1. Patient characteristics of the training set and the SEER validation set

\begin{tabular}{|c|c|c|}
\hline \multirow[t]{2}{*}{ Variable } & Training set $(\mathrm{N}=5,206)$ & Validation $\operatorname{set}(\mathrm{N}=1,986)$ \\
\hline & Mean (SD)/N (\%) & Mean (SD)/N (\%) \\
\hline \multicolumn{3}{|l|}{ Year of diagnosis } \\
\hline 2002-2004 & $3,251(62.5)$ & - \\
\hline 2005-2006 & 1,955 (37.5) & - \\
\hline 2007-2008 & - & $1,986(100)$ \\
\hline Age, years & $65.1(13.4)$ & $64.6(13.5)$ \\
\hline \multicolumn{3}{|l|}{ Race } \\
\hline White & $3,501(67.2)$ & $1,292(65.1)$ \\
\hline Black & $677(13.0)$ & $267(13.4)$ \\
\hline Other & $1,018(19.6)$ & $425(21.4)$ \\
\hline Unknown & $10(0.2)$ & $2(1.0)$ \\
\hline \multicolumn{3}{|l|}{ Gender } \\
\hline Female & $1,979(38.0)$ & $731(36.8)$ \\
\hline Male & $3,227(62.0)$ & $1,255(63.2)$ \\
\hline \multicolumn{3}{|l|}{ Tumor location } \\
\hline Cardia & $1,417(27.2)$ & $567(28.5)$ \\
\hline Upper one-third & $143(2.7)$ & $164(8.3)$ \\
\hline Middle one-third & $379(7.3)$ & $60(3.0)$ \\
\hline Lower one-third & $1,565(30.1)$ & $598(30.1)$ \\
\hline Not specified & $1,702(32.7)$ & $597(30.1)$ \\
\hline Tumor size, $\mathrm{cm}$ & $5.4(3.2)$ & $5.4(4.3)$ \\
\hline Unknown & $1,054(20.2)$ & $239(12.0)$ \\
\hline \multicolumn{3}{|l|}{ Tumor differentiation } \\
\hline Poorly or undifferentiated & 3,617 (69.5) & $1,395(70.2)$ \\
\hline $\begin{array}{l}\text { Well or moderately } \\
\text { differentiated }\end{array}$ & $1,406(27.0)$ & $521(26.2)$ \\
\hline Unknown & $183(3.5)$ & $70(3.5)$ \\
\hline \multicolumn{3}{|l|}{ T stage } \\
\hline $\mathrm{T} 1$ & $253(4.9)$ & $115(5.8)$ \\
\hline $\mathrm{T} 2$ & $824(15.8)$ & $306(15.4)$ \\
\hline $\mathrm{T} 3$ & $1,980(38.0)$ & $865(43.6)$ \\
\hline $\mathrm{T} 4 \mathrm{a}$ & $1,613(31.0 \%)$ & $542(27.3)$ \\
\hline $\mathrm{T} 4 \mathrm{~b}$ & $536(10.3)$ & $158(8.0)$ \\
\hline Mean number of MLNs & $4.9(6.5)$ & $4.7(6.5)$ \\
\hline \multicolumn{3}{|l|}{$\mathrm{N}$ stage } \\
\hline No & $1,418(27.2)$ & $558(28.1)$ \\
\hline N1 & $1,221(23.5)$ & $491(24.7)$ \\
\hline N2 & $1,197(23.0)$ & $435(21.9)$ \\
\hline N3a & $1,009(19.4)$ & $377(19.0)$ \\
\hline N3b & $361(6.9)$ & $125(6.3)$ \\
\hline Mean number of NLNs & $10.7(10.3)$ & $12.7(11.2)$ \\
\hline LNR & $0.33(0.33)$ & $0.29(0.31)$ \\
\hline \multicolumn{3}{|l|}{ AJCC stage } \\
\hline IB & $632(12.1)$ & $236(11.9)$ \\
\hline IIA & $786(15.1)$ & $357(18.0)$ \\
\hline IIB & 898 (17.2) & $352(17.7)$ \\
\hline IIIA & $875(16.8)$ & $319(16.1)$ \\
\hline IIIB & $1,098(21.1)$ & $455(22.9)$ \\
\hline IIIC & 917 (17.6) & $267(13.4)$ \\
\hline \multicolumn{3}{|l|}{ Receipt of ART } \\
\hline Yes & $2,449(47.0)$ & $933(47.0)$ \\
\hline No & $2,757(53.0)$ & $1,053(53.0)$ \\
\hline
\end{tabular}

SEER, Surveillance, Epidemiology, and End Results database; SD, standard deviation; MLN, metastatic lymph node; NLN, negative lymph node; LNR, lymph node ratio; AJCC, American Joint Committee on Cancer; ART, adjuvant radiotherapy.

A nomogram to predict the 3- and 5-year OS was developed from the final model (Figure 2). The unadjusted C-index was 0.68 (95\% CI, 0.67-0.69), which was superior to that of the $8^{\text {th }}$ AJCC TNM classification $(0.65 ; 95 \% \mathrm{CI}, 0.64-0.66 ; P<0.01)$. The bootstrap-adjusted C-index remained at 0.68 , which 
indicated minimal evidence of model overfit. The calibration plots showed close agreement between the actual OS probabilities in the patients in the training set and the OS predicted from the nomogram (Supplementary Figures 1a and 1b).

Table 2. Selected prognosticators according to the multivariate Cox regression analysis weighted by inverse propensity score

\begin{tabular}{|c|c|c|c|}
\hline \multirow[t]{2}{*}{ Variable } & \multicolumn{3}{|c|}{ Multivariate Cox model } \\
\hline & HR & $95 \% \mathrm{CI}$ & $P$ value \\
\hline Agea & $4.81^{\mathrm{a}}$ & $4.35-5.81$ & $<0.01$ \\
\hline \multicolumn{4}{|l|}{ Tumor location } \\
\hline Lower one-third & 1 & & \\
\hline Middle one-third & 1.05 & $0.91-1.20$ & 0.52 \\
\hline Upper one-third & 1.36 & $1.07-1.72$ & 0.01 \\
\hline Cardia & 1.35 & $1.23-1.49$ & $<0.01$ \\
\hline \multicolumn{4}{|l|}{ Tumor differentiation } \\
\hline Poorly or undifferentiated & 1 & & \\
\hline Well or moderately differentiated & 0.91 & $0.84-1.00$ & 0.04 \\
\hline \multicolumn{4}{|l|}{ Race } \\
\hline White & 1 & & \\
\hline Black & 1.16 & $1.02-1.32$ & 0.02 \\
\hline Other & 0.91 & $0.81-1.01$ & 0.09 \\
\hline \multicolumn{4}{|l|}{ T stage } \\
\hline $\mathrm{T} 1$ & 1 & & \\
\hline $\mathrm{T} 2$ & 1.34 & $1.07-1.67$ & 0.01 \\
\hline $\mathrm{T} 3$ & 1.75 & $1.43-2.15$ & $<0.01$ \\
\hline T4a & 2.27 & $1.85-2.79$ & $<0.01$ \\
\hline $\mathrm{T} 4 \mathrm{~b}$ & 3.10 & $2.46-3.90$ & $<0.01$ \\
\hline $\mathrm{LNR}^{\mathrm{b}}$ & - & - & $<0.01$ \\
\hline Receipt of ART ${ }^{b}$ & - & - & 0.36 \\
\hline Interaction term (LNR and receipt of ART) ${ }^{b}$ & - & - & $<0.01$ \\
\hline \multicolumn{4}{|l|}{ Receipt of ART by LNR b } \\
\hline \multicolumn{4}{|l|}{$\mathrm{LNR}=0$} \\
\hline No & 1 & & \\
\hline Yes & 1.13 & $0.88-1.45$ & 0.34 \\
\hline \multicolumn{4}{|l|}{$0<\mathrm{LNR} \leq 0.2$} \\
\hline No & 1 & & \\
\hline Yes & 0.93 & $0.78-1.11$ & 0.45 \\
\hline \multicolumn{4}{|l|}{$0.2<\mathrm{LNR} \leq 0.5$} \\
\hline No & 1 & & \\
\hline Yes & 0.70 & $0.60-0.82$ & $<0.01$ \\
\hline \multicolumn{4}{|l|}{ LNR $>0.5$} \\
\hline No & 1 & & \\
\hline Yes & 0.78 & $0.69-0.89$ & $<0.01$ \\
\hline \multicolumn{4}{|l|}{ LNR by receipt of $A R T^{b}$} \\
\hline \multicolumn{4}{|l|}{ Receiving ART } \\
\hline LNRa & 7.39a & $6.96-8.33$ & $<0.01$ \\
\hline \multicolumn{4}{|l|}{ Not receiving ART } \\
\hline LNR & 3.32 & $2.83-3.60$ & $<0.01$ \\
\hline
\end{tabular}

HR, hazard ratio; 95\% CI, 95\% confidence interval; LNR, lymph node ratio; ART, adjuvant radiotherapy.

a. These variables had non-linear effects on the log hazards of survival and were

transformed to appropriate forms, namely $(\text { age } / 100)^{3}$ and $\sqrt{\mathrm{LNR}+0.1}$ (for patients with ART).

b. HRs for LNR and receipt of ART are not shown because a significant interaction was found between the two variables, which indicates that the HRs for the receipt of ART differ according to LNR, whereas the HRs for LNR vary by receipt of ART. Accordingly, the HRs for the receipt of ART at different LNR levels and the HRs for LNR by receipt of ART are presented.

The C-index was 0.68 (95\% CI, 0.67-0.69) when applied to the weighted SEER validation set. The nomogram showed significantly greater discriminatory power than the $8^{\text {th }}$ AJCC TNM classification in the validation set (C-index: $0.64 ; 95 \%$ CI, $0.63-0.65 ; P<0.01)$. Calibration was consistent between the actual probabilities and the nomogram-predicted OS among the validation sets (Supplementary Figures 1c-1d).

In both the training set and the validation set, the nomogram-estimated survival benefit from ART was significantly predictive of the magnitude of improved OS among patients treated with ART ( $P_{\text {interaction }}<0.01$ and $P_{\text {interaction }}=0.02$, respectively). In both datasets, ART demonstrated no significant improvement of OS among patients with a lower estimated survival benefit (below or equal to the median values) compared with patients with a higher estimated survival benefit (above the median values), who saw an approximate $20 \%-30 \%$ reduction in the hazard of death (Figures 3a-3b).

Supplementary Figure 2 shows the Web software that predicts the 3- and 5-year OS probabilities with or without ART. These predictions can be calculated by entering clinicopathologic variables on the website, rather than drawing lines and adding up points in the nomogram.

\section{Discussion}

Internationally, there is a goal toward better risk stratification and optimization of adjuvant treatment strategies for patients with resected GC. Subgroup analyses in phase III trials have suggested that the benefit from ART \pm chemotherapy may vary according to certain clinicopathologic factors (e.g., lymph node status, Lauren's type) [19, 20]. However, these findings are hypothesis-generating and require verification in future clinical trials [21]. Furthermore, important differences may exist between patients treated in the clinical trial setting and those treated in the general clinical practice, particularly regarding patient demographic characteristics and treatment quality. [22-24] Combined, there is a need for effective patient selection criteria for ART in the general clinical practice.

In this population-based study, we developed and validated a nomogram that was capable of providing individualized estimates of the potential survival benefit from ART. The efficacy of ART was positively correlated with the estimated benefits of ART; that is, the nomogram can aid individualized management decisions in the use of ART for GC. For example, a 70-year-old (38 points) Caucasian patient (7 points) with pT2 (13 points) poorly differentiated (7 points) lower one-third GC (4 points) who received ART following surgery with a total of 20 nodes harvested and 2 positive nodes (LNR, $0.1 ; 11$ points) would have a corresponding 5-year OS of 57\% (80 points). If this patient did not receive ART, the 
predicted 5 -year OS would be 55\% (86 points). For patients with estimates of survival benefit $\leq 4 \%$, the efficacy of ART was minimal, and the use of ART might be dispensable in this patient.

Development of prognostic nomograms is currently a hot topic. A number of prognostic nomograms for patients with resected GC have been developed recently, with over 20 published between June 2016 and May 2017 [25-45]. However, few of the published nomograms have been tested the ability to optimize treatment strategies for patients with resected GC: In our previous study, we developed a nomogram which is capable of predicting the individual survival benefit of adjuvant 5-fluroracil plus oxaliplatin [46]; additionally, Jiang et al. developed two nomograms to provide individualized survival estimates for patients treated with and without adjuvant chemotherapy, respectively [25]. To the best of our knowledge, the present nomogram is the first one to provide guidance for the individualized use of ART, which is preferred in the US.

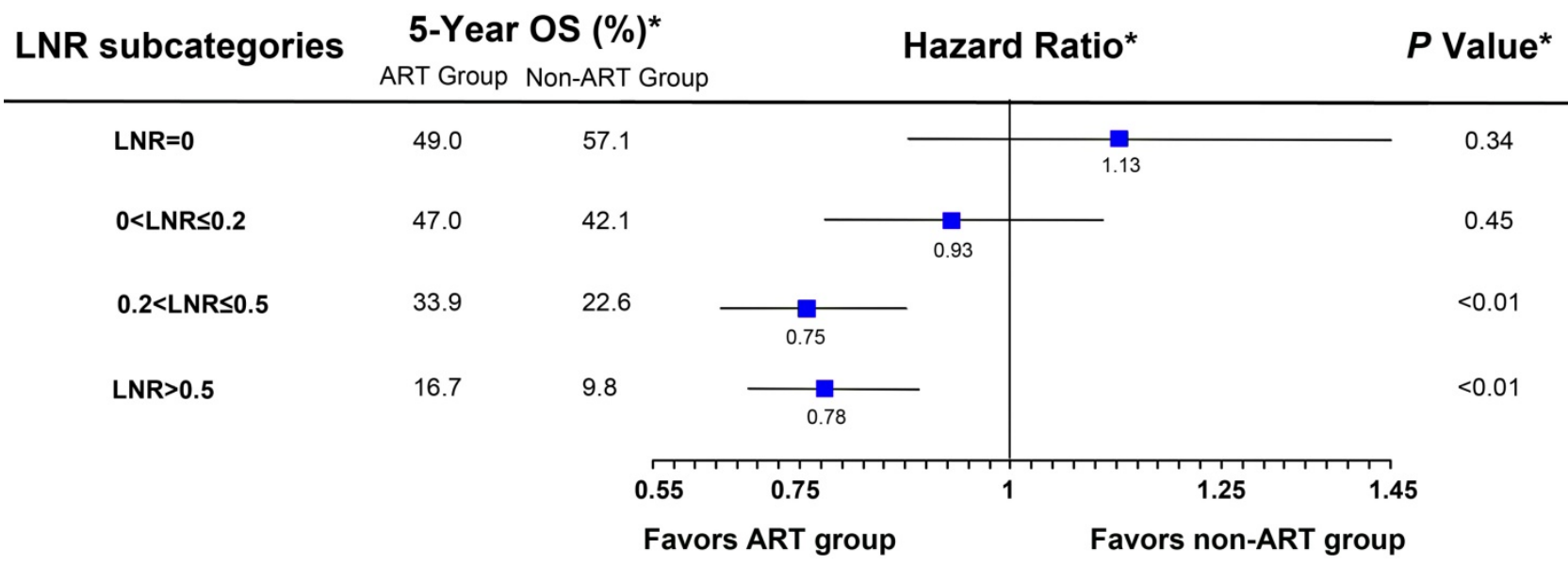

Figure 1. Hazard ratio comparing overall survival (OS) between the adjuvant radiotherapy (ART) group and the non-ART group in the training set according to the lymph node ratio (LNR). Patients were stratified based on the quartiles of LNR. $(*)$ Weighted by inverse propensity score.

Points

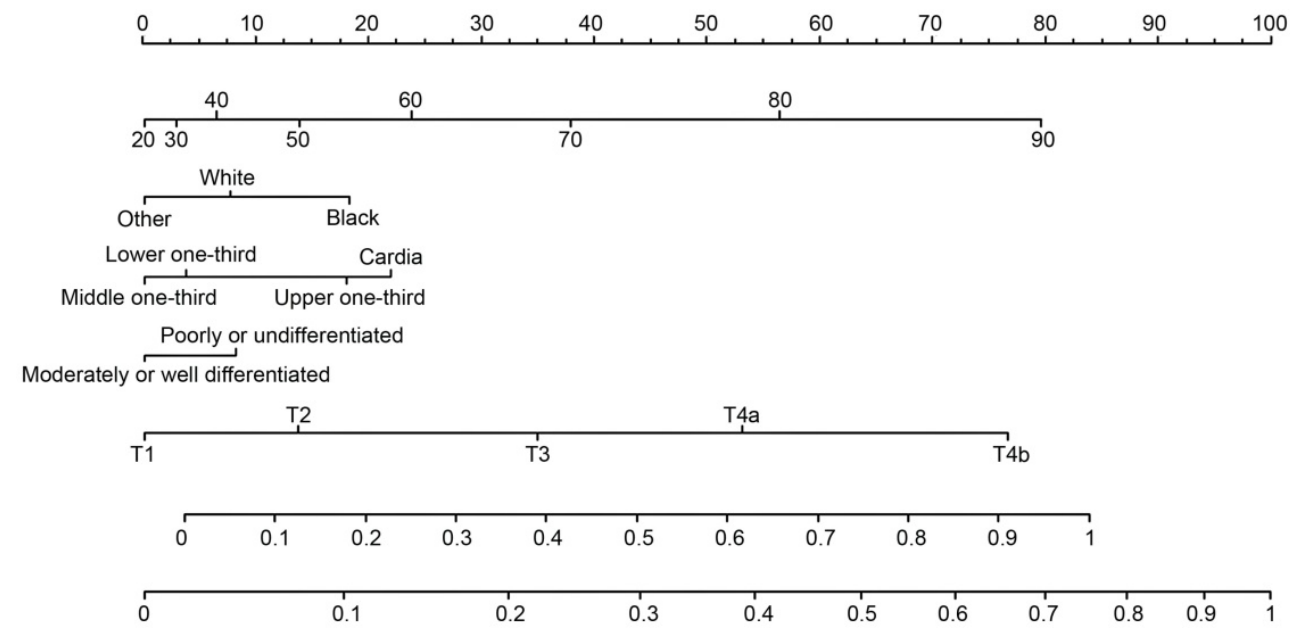

Age, years

Race

Tumor location

Tumor differentiation

T stage

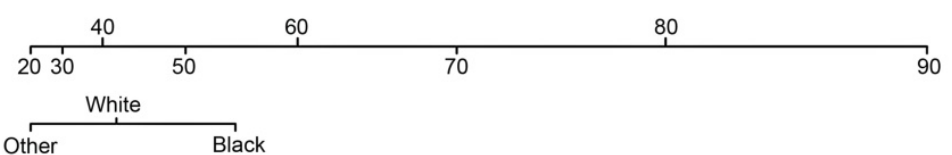

$\left[\begin{array}{l}\text { LNR (with ART) } \\ \text { LNR (without ART) }\end{array}\right.$

Total points

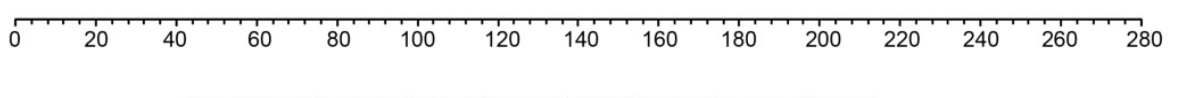

3-year OS

5-year OS

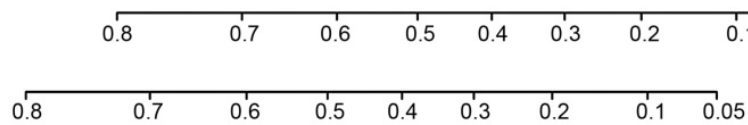

Figure 2. Nomogram for predicting 3-and 5-year overall survival (OS) in patients with resected gastric cancer. The nomogram is generated by adding the points projected on the points scale by each variable. The total points projected on the bottom scales indicate the estimated 3-and 5-year OS probabilities. For the lymph node ratio (LNR) scales, the points assigned are based on whether the patient received adjuvant radiotherapy (ART). For example, if a patient received ART and had an LNR of 0 , then 0 points are given for the LNR scales. By contrast, if a patient did not receive ART and had an LNR of 0.4, then 49 points are given for the LNR scales. 
(a)

\begin{tabular}{|c|c|c|c|c|c|}
\hline \multirow{3}{*}{$\begin{array}{c}\text { Estimated Survival Benefit } \\
\leq 0\end{array}$} & \multicolumn{2}{|c|}{5 -Year OS $(\%)^{*}$} & \multirow{2}{*}{\multicolumn{2}{|c|}{ Hazard Ratios* }} & \multirow{2}{*}{$P$ Value* } \\
\hline & ART Group & Non-ART Group & & & \\
\hline & 49.8 & 56.7 & & $\overrightarrow{1.13}$ & 0.35 \\
\hline$>0$ and $\leq 4 \%$ & 32.2 & 31.6 & $\rightarrow$ & & 0.21 \\
\hline$>4 \%$ and $\leq 8 \%$ & 38.4 & 32.3 & $\overrightarrow{0.82}$ & & $<0.01$ \\
\hline$>8 \%$ & 36.3 & 26.6 & $\overrightarrow{0.75}$ & & $<0.01$ \\
\hline & & 0. & & & \\
\hline & & Favo & RT group & Fav & T group \\
\hline
\end{tabular}

(b)

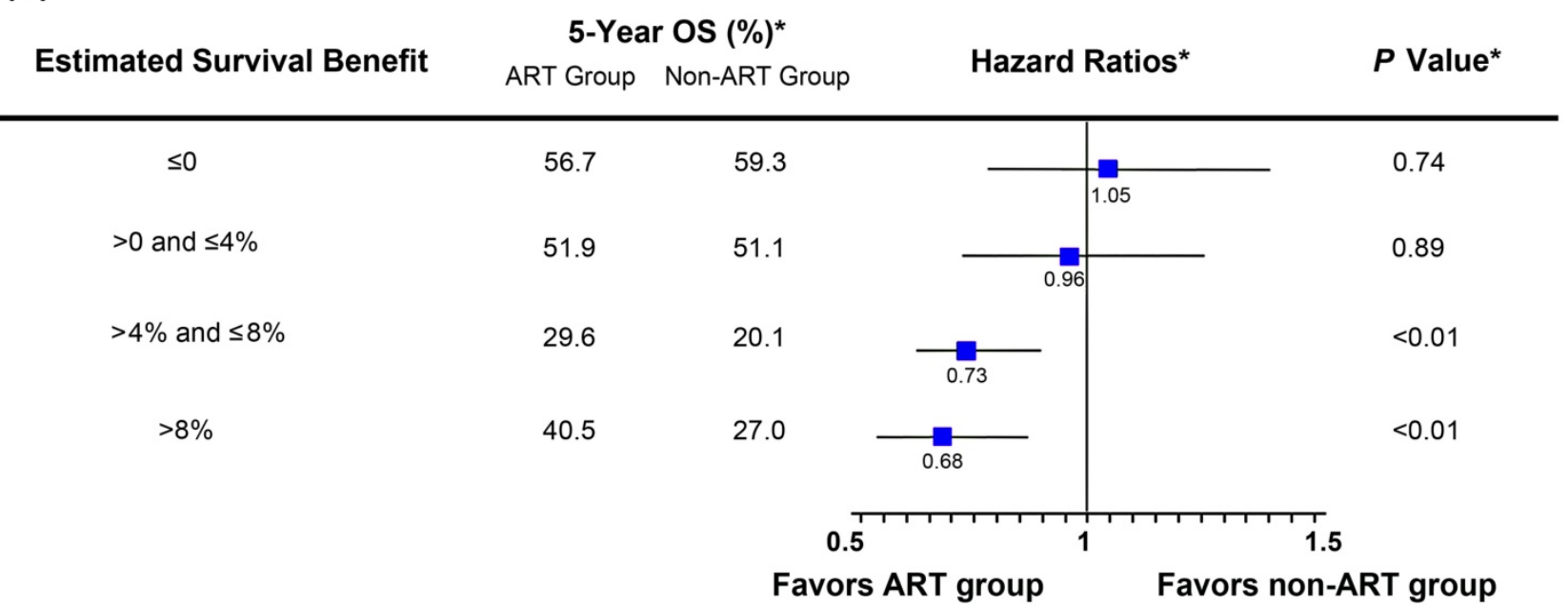

Figure 3. Hazard ratio comparing overall survival (OS) between the adjuvant radiotherapy (ART) group and the non-ART group according to the nomogram-estimated survival benefit from ART. The estimated survival benefit of ART was calculated as the difference in the nomogram-predicted 5 -year OS rates between the ART and non-ART groups. Patients were stratified based on the quartiles of the estimated survival benefit of ART. In both the training set (a) and the validation set (b), the nomogram-estimated survival benefit from ART was positively associated with the magnitude of improved OS among patients treated with ART. (*) Weighted by inverse propensity score.

The current study has several strengths in terms of methodological approaches and clinical usefulness. Compared with Jiang's study [25], we adopted the multivariable fractional polynomial interaction model which is capable of deriving a unified prognostic nomogram instead of two nomograms for patients treated with and without adjuvant treatment, respectively. Additionally, we used LNR to refine the prognostic accuracy of lymph node staging. As demonstrated in our previous study, LNR is as powerful as and more simplified than the log odds of positive lymph nodes (LODDS) when included as a prognostic indicator in nomograms for patients with resected GC.[28] Moreover, as compared with the previous nomograms, almost all of which were derived from data of high-volume institutions, the current nomogram was developed using a US population-based cohort, which reflects the routine management of GC in the general US population and suggests the potential generalizability of the nomogram. Even though the SEER cohort represents the community standards, the magnitude of improved discrimination of the current nomogram over the TNM staging ( $\Delta$ C-statistic, 0.03 , and 0.04 in the training set and the validation set, respectively) compared favorably with the previous US nomograms (ranging from 0.01 to 0.03 ). [9, 47-50]

Although ART significantly improved survival in patients with high predictions of survival benefit, the outcomes in the ART arm were unsatisfactory, 
suggesting a room for improvement. Currently, the optimal modality of treatment in patients with resectable locally advanced GC remains unsettled. The ongoing TOPGEAR trial was designed to evaluate the efficacy of adding neoadjuvant chemoradiation to perioperative chemotherapy in patients undergoing curative surgery. [51] Additionally, in light of the positive results from the ToGA trial in advanced GC, [52] two ongoing phase II trials were conducted to assess the safety, tolerability and efficacy of the adding trastuzumab to chemo(radio)therapy in the neoadjuvant and adjuvant setting. [53,54] The results of these trials will be valuable in improving the treatment modalities and survival outcomes in patients with locally advanced GC.

The present study has several limitations. First, SEER does not provide chemotherapy data. However, other US population-based data suggested that there were only small fractions of patients who received ART without chemotherapy $(<10 \%)$ or received chemotherapy without ART $(10 \%-20 \%)$, regardless before and after 2006. [55, 56] Moreover, although there was solid evidence for the use of perioperative chemotherapy without irradiation after 2006, the proportions of patients receiving ART were similar before and after 2006 (Table 1).Taken together, these findings suggest that adjuvant chemoradiotherapy remains the mainstream in the adjuvant setting in the US while the use of chemotherapy alone without radiation is uncommon. Thus, we believe that the proposed nomogram actually predicts the survival benefit of adjuvant chemoradiotherapy over surgery alone. More importantly, the proposed nomogram retained sufficient discriminatory power and accurate calibration for the patients diagnosed after 2006. Second, other unavailable factors such as marginal status, medical comorbidities and performance status may also influence the overall results. These additional factors should be taken into account when evaluating the use of ART. Finally, further validation of the nomogram using non-US cohorts is required.

In summary, we developed and validated a nomogram to predict the 3- and 5-year OS with and without ART among patients with resected GC. We also developed accompanying Web software to facilitate the use of the nomogram without cumbersome procedures. The proposed nomogram and Web software, which uses readily available clinicopathologic factors, will be clinically useful to patients and clinicians when evaluating adjuvant treatment options.

\section{Abbreviation}

GC: Gastric cancer; ART: adjuvant radiotherapy;
AJCC: American Joint Committee on Cancer; OS: overall survival; SEER: Surveillance, Epidemiology, and End Results; LNR: lymph node ratio.

\section{Supplementary Material}

Supplementary figures and tables. http://www.jcancer.org/v08p3498s1.pdf

\section{Acknowledgements}

We thank the staff members of the National Cancer Institute and their colleagues across the United States and at Information Management Services, Inc., who have been involved with the Surveillance, Epidemiology, and End Results (SEER) Program.

\section{Funding}

This work was supported by:

1. National High Technology Research and Development Program of China (863 Program), China (No. 2015AA020103).

2. National Natural Science Foundation of China (No.81372570, 81572392).

3. Science and Technology Program of Guangdong Province, China (No. 201508020247).

4. Medical Scientific Research of Guangdong province (No. B201416).

5. China Scholarship Council

(No. 201406385034).

6. Science and Technology Planning Project of Guangdong Province (No. 2013A022100023).

\section{Competing Interests}

The authors have declared that no competing interest exists.

\section{References}

1. Torre LA, Bray F, Siegel RL, Ferlay J, Lortet-Tieulent J, Jemal A. Global cancer statistics, 2012. CA Cancer J Clin. 2015; 65: 87-108.

2. Chen $W$, Zheng R, Zeng H, Zhang S. The updated incidences and mortalities of major cancers in China, 2011. Chin J Cancer. 2015; 34: 53.

3. Chen W, Zheng R, Baade PD, Zhang S, Zeng H, Bray F, et al. Cancer statistics in China, 2015. CA Cancer J Clin. 2016; 66: 115-32.

4. Macdonald JS, Smalley SR, Benedetti J, Hundahl SA, Estes NC, Stemmermann GN, et al. Chemoradiotherapy after surgery compared with surgery alone for adenocarcinoma of the stomach or gastroesophageal junction. N Engl J Med. 2001; 345: 725-30.

5. Cunningham D, Allum WH, Stenning SP, Thompson JN, Van de Velde CJ, Nicolson $M$, et al. Perioperative chemotherapy versus surgery alone for resectable gastroesophageal cancer. N Engl J Med. 2006; 355: 11-20.

6. Bang YJ, Kim YW, Yang HK, Chung HC, Park YK, Lee KH, et al. Adjuvant capecitabine and oxaliplatin for gastric cancer after D2 gastrectomy (CLASSIC): a phase 3 open-label, randomised controlled trial. Lancet (London, England). 2012; 379: 315-21.

7. Lee J, Lim do H, Kim S, Park SH, Park JO, Park YS, et al. Phase III trial comparing capecitabine plus cisplatin versus capecitabine plus cisplatin with concurrent capecitabine radiotherapy in completely resected gastric cancer with D2 lymph node dissection: the ARTIST trial. J Clin Oncol. 2012; 30: 268-73.

8. [Internet] National Comprehensive Cancer Network (NCCN). NCCN clinical practice guidelines in oncology (NCCN guidelines): gastric cancer; 2016. http://www.nccn.org/professionals/physician_gls/f_guidelines_nojava.asp [accessed January 15, 2016] 
9. Kattan MW, Karpeh MS, Mazumdar M, Brennan MF. Postoperative nomogram for disease-specific survival after an R0 resection for gastric carcinoma. J Clin Oncol. 2003; 21: 3647-50.

10. Han DS, Suh YS, Kong SH, Lee HJ, Choi Y, Aikou S, et al. Nomogram predicting long-term survival after $\mathrm{d} 2$ gastrectomy for gastric cancer. J Clin Oncol. 2012; 30: 3834-40.

11. Hirabayashi S, Kosugi S, Isobe Y, Nashimoto A, Oda I, Hayashi K, et al. Development and external validation of a nomogram for overall survival after curative resection in serosa-negative, locally advanced gastric cancer. Ann Oncol. 2014; 25: 1179-84.

12. Stekhoven DJ, Buhlmann P. MissForest--non-parametric missing value imputation for mixed-type data. Bioinformatics. 2012; 28: 112-8.

13. Austin PC. The performance of different propensity score methods for estimating marginal hazard ratios. Stat Med. 2013; 32: 2837-49.

14. Lin DY, Wei LJ. The robust inference for the Cox proportional hazards model. J Am Stat Assoc. 1989; 84: 1074-8.

15. Royston $\mathrm{P}$, Sauerbrei $\mathrm{W}$. Interaction of treatment with a continuous variable: simulation study of significance level for several methods of analysis. Stat Med. 2013; 32: 3788-803.

16. Wang $\mathrm{W}, \mathrm{Xu} \mathrm{DZ}, \mathrm{Li} \mathrm{YF}$, Guan $\mathrm{YX}$, Sun $\mathrm{XW}$, Chen $\mathrm{YB}$, et al. Tumor-ratio-metastasis staging system as an alternative to the 7 th edition UICC TNM system in gastric cancer after D2 resection--results of a single-institution study of 1343 Chinese patients. Ann Oncol. 2011; 22: 2049-56.

17. Harrell FE, Jr., Lee KL, Mark DB. Multivariable prognostic models: issues in developing models, evaluating assumptions and adequacy, and measuring and reducing errors. Stat Med. 1996; 15: 361-87.

18. Iasonos A, Schrag D, Raj GV, Panageas KS. How to build and interpret a nomogram for cancer prognosis. J Clin Oncol. 2008; 26: 1364-70.

19. Smalley SR, Benedetti JK, Haller DG, Hundahl SA, Estes NC, Ajani JA, et al. Updated analysis of SWOG-directed intergroup study 0116: a phase III trial of adjuvant radiochemotherapy versus observation after curative gastric cancer resection. J Clin Oncol. 2012; 30: 2327-33.

20. Park SH, Sohn TS, Lee J, Lim do H, Hong ME, Kim KM, et al. Phase III Trial to Compare Adjuvant Chemotherapy With Capecitabine and Cisplatin Versus Concurrent Chemoradiotherapy in Gastric Cancer: Final Report of the Adjuvant Chemoradiotherapy in Stomach Tumors Trial, Including Survival and Subset Analyses. J Clin Oncol. 2015; 33: 3130-6.

21. Goodman KA. Refining the Role for Adjuvant Radiotherapy in Gastric Cancer: Risk Stratification Is Key. J Clin Oncol. 2015; 33: 3082-4.

22. Murthy VH, Krumholz HM, Gross CP. Participation in cancer clinical trials: race-, sex-, and age-based disparities. JAMA. 2004; 291: 2720-6.

23. Britton A, McKee M, Black N, McPherson K, Sanderson C, Bain C. Threats to applicability of randomised trials: exclusions and selective participation. J Health Serv Res Policy. 1999; 4: 112-21.

24. Dikken JL, Wouters MW, Lemmens VE, Putter H, van der Geest LG, Verheij $\mathrm{M}$, et al. Influence of hospital type on outcomes after oesophageal and gastric cancer surgery. Br J Surg. 2012; 99: 954-63.

25. Jiang Y, Zhang Q, Hu Y, Li T, Yu J, Zhao L, et al. ImmunoScore Signature: A Prognostic and Predictive Tool in Gastric Cancer. Ann Surg. 2016.

26. Peng P, Min L, Song S, Zhao J, Li L, Yang C, et al. Elevated Expression of Calpain-4 Predicts Poor Prognosis in Patients with Gastric Cancer after Gastrectomy. Int J Mol Sci. 2016; 17.

27. Muneoka Y, Akazawa K, Ishikawa T, Ichikawa H, Nashimoto A, Yabusaki H, et al. Nomogram for 5-year relapse-free survival of a patient with advanced gastric cancer after surgery. Int J Surg. 2016; 35: 153-9.

28. Wang ZX, Qiu MZ, Jiang YM, Zhou ZW, Li GX, Xu RH. Comparison of prognostic nomograms based on different nodal staging systems in patients with resected gastric cancer. J Cancer. 2017; 8: 950-8.

29. Zhao J, Li H, Min L, Han X, Shu P, Yang Y, et al. High expression of Tumor necrosis factor receptor-associated factor 2 promotes tumor metastasis and is associated with unfavorable prognosis in gastric cancer. J Gastroenterol Hepatol. 2017.

30. Wang PL, Xiao FT, Gong BC, Liu FN, Xu HM. A Nomogram for Predicting Overall Survival of Gastric Cancer Patients with Insufficient Lymph Nodes Examined. J Gastrointest Surg. 2017.

31. Cao Y, Liu H, Zhang H, Lin C, Li R, Wu S, et al. CXC chemokine receptor 1 predicts postoperative prognosis and chemotherapeutic benefits for TNM II and III resectable gastric cancer patients. Oncotarget. 2017; 8: 20328-39.

32. Chen S, Rao H, Liu J, Geng Q, Guo J, Kong P, et al. Lymph nodes ratio based nomogram predicts survival of resectable gastric cancer regardless of the number of examined lymph nodes. Oncotarget. 2017.

33. Chen XL, Chen XZ, Wang YG, He D, Lu ZH, Liu K, et al Clinical significance of putative markers of cancer stem cells in gastric cancer: A retrospective cohort study. Oncotarget. 2016; 7: 62049-69.

34. Li X, Zhang W, Zhang X, Wang H, Xu K, Yao H, et al. The prognostic value of negative lymph node count for patients with gastric cancer who received preoperative radiotherapy. Oncotarget. 2017

35. Lin $\mathrm{C}$, Liu $\mathrm{H}$, Zhang $\mathrm{H}, \mathrm{He} \mathrm{H}, \mathrm{Li} \mathrm{H}$, Shen $\mathrm{Z}$, et al. Interleukin-13 receptor alpha2 is associated with poor prognosis in patients with gastric cancer after gastrectomy. Oncotarget. 2016; 7: 49281-8.

36. Lin JP, Lin JX, Cao LL, Zheng CH, Li P, Xie JW, et al. Preoperative lymphocyte-to-monocyte ratio as a strong predictor of survival and recurrence for gastric cancer after radical-intent surgery. Oncotarget. 2017.

37. Liu J, Geng Q, Chen S, Liu X, Kong P, Zhou Z, et al. Nomogram based on systemic inflammatory response markers predicting the survival of patients with resectable gastric cancer after D2 gastrectomy. Oncotarget. 2016; 7: 37556-65.

38. Liu J, Geng Q, Liu Z, Chen S, Guo J, Kong P, et al. Development and external validation of a prognostic nomogram for gastric cancer using the national cancer registry. Oncotarget. 2016; 7: 35853-64.

39. Sun H, He B, Nie Z, Pan Y, Lin K, Peng H, et al. A nomogram based on serum bilirubin and albumin levels predicts survival in gastric cancer patients. Oncotarget. 2017.

40. Wang W, Chen XL, Zhao SY, Xu YH, Zhang WH, Liu K, et al. Prognostic significance of preoperative serum CA125, CA19-9 and CEA in gastric carcinoma. Oncotarget. 2016; 7: 35423-36.

41. Wang ZX, Yang LP, Qiu MZ, Wang ZQ, Zhou YX, Wang F, et al. Prognostic value of preoperative serum lactate dehydrogenase levels for resectable gastric cancer and prognostic nomograms. Oncotarget. 2016; 7: 39945-56.

42. Zhao LY, Chen XL, Wang YG, Xin Y, Zhang WH, Wang YS, et al. A new predictive model combined of tumor size, lymph nodes count and lymphovascular invasion for survival prognosis in patients with lymph node-negative gastric cancer. Oncotarget. 2016; 7: 72300-10.

43. Zheng WF, Ji TT, Lin Y, Li RZ. The prognostic value of lymph nodes count on survival of patients with node-negative gastric cancer. Oncotarget. 2016; 7: 43680-8.

44. Cao Y, Zhang H, Liu H, Lin C, Li R, Wu S, et al. Glycoprotein 130 is associated with adverse postoperative clinical outcomes of patients with late-stage non-metastatic gastric cancer. Sci Rep. 2016; 6: 38364.

45. Wu S, Liu H, Zhang H, Lin C, Li R, Cao Y, et al. Galectin-8 is associated with recurrence and survival of patients with non-metastatic gastric cancer after surgery. Tumour Biol. 2016; 37: 12635-42.

46. Wang ZX, Yang XL, He MM, Wang F, Zhang DS, Li YH, et al. The Efficacy of Adjuvant FOLFOX6 for Patients With Gastric Cancer after D2 Lymphadenectomy: A Propensity Score-matched Analysis. Medicine (Baltimore). 2016; 95: e3214.

47. Kim Y, Spolverato G, Ejaz A, Squires MH, Poultsides G, Fields RC, et al. A nomogram to predict overall survival and disease-free survival after curative resection of gastric adenocarcinoma. Ann Surg Oncol. 2015; 22: 1828-35.

48. Dikken JL, Baser RE, Gonen M, Kattan MW, Shah MA, Verheij M, et al. Conditional probability of survival nomogram for 1-, 2-, and 3-year survivors after an R0 resection for gastric cancer. Ann Surg Oncol. 2013; 20: 1623-30.

49. Novotny AR, Schuhmacher C, Busch R, Kattan MW, Brennan MF, Siewert JR. Predicting individual survival after gastric cancer resection: validation of a US-derived nomogram at a single high-volume center in Europe. Ann Surg. 2006; 243: 74-81.

50. Peeters KC, Kattan MW, Hartgrink HH, Kranenbarg EK, Karpeh MS, Brennan $\mathrm{MF}$, et al. Validation of a nomogram for predicting disease-specific survival after an R0 resection for gastric carcinoma. Cancer. 2005; 103: 702-7.

51. Trial of preoperative therapy for gastric and esophagogastric junction adenocarcinoma (TOPGEAR). Available from URL: https://clinicaltrials.gov/ ct2/show/NCT01924819 [accessed February 11, 2016].

52. Bang YJ, Van Cutsem E, Feyereislova A, Chung HC, Shen L, Sawaki A, et al. Trastuzumab in combination with chemotherapy versus chemotherapy alone for treatment of HER2-positive advanced gastric or gastro-oesophageal junction cancer (ToGA): a phase 3, open-label, randomised controlled trial. Lancet. 2010; 376: 687-97.

53. [Internet] Neoadjuvant study using trastuzumab or trastuzumab with pertuzumab in gastric or gastroesophageal junction adenocarcinoma (INNOVATION). https://clinicaltrials.gov/ct2/show/NCT02205047.

54. [Internet] A study of the combination of oxaliplatin, capecitabine and herceptin (Trastuzumab) and chemoradiotherapy in the adjuvant setting in operated patients with HER2+ gastric or gastro-esophageal junction cancer (TOXAG study). https://clinicaltrials.gov/ct2/show/NCT01748773.

55. Stessin AM, Sherr DL. Demographic disparities in patterns of care and survival outcomes for patients with resected gastric adenocarcinoma. Cancer Epidemiol Biomarkers Prev. 2011; 20: 223-33.

56. Datta J, McMillan MT, Shang EK, Mamtani R, Lewis RS, Jr., Kelz RR, et al. Omission of adjuvant therapy after gastric cancer resection: development of a validated risk model. J Natl Compr Canc Netw. 2015; 13: 531-41. 